\title{
Applying Model Order Reduction to the Reliability Prediction of Power Electronic Module Wirebond Structure
}

\author{
Pushpa Rajaguru, Martina Bella, and Chris Bailey \\ Computational Mechanics Reliability Group, University of Greenwich \\ London, United Kingdom \\ Telephone: +44(0)2083318445, e-mail:p.rajaguru@gre.ac.uk
}

\begin{abstract}
Predicting the reliability of power electronics module wirebond structures requires accurate computer models to investigate the design space constraints in a computationally efficient manner. This paper details a model-order reduction (MOR) method to solve the governing equations for electro-thermal behaviour of wire-bond structures and a linear-damage rule and fatigue model to predict their wear-out behaviour. Various MOR methods are compared in terms of their accuracy and computational efficiency. Finite element calculations are used to validate the MOR predictions in terms of accuracy and solution times. The paper presents for the first time the significant benefits that MOR techniques can provide to reliability engineers for predicting the electro-thermal and fatigue behaviour of wirebonds in power modules. For the six MOR methods assessed, the Rational Krylov Algorithm (RKA) outperforms all other MOR methods in terms of accuracy and solution times, where it provides a solution 84 times faster than a full finite element solver.
\end{abstract}

\section{INTRODUCTION}

Power electronics modules (PEM) play a vital role in the conversion, control of alternative energy generation, and distribution. All power devices, such as IGBT, have finite onstate voltage drops during turn-on and turn-off and this causes a small amount of power to be dissipated and converted into heat energy within the PEM device. Combination of converted heat energy and the environment temperature can initiate thermomechanical failure mechanisms within the device due to the coefficients of thermal expansion (CTE) mismatch between various materials of the components. The wirebond lift-off is one of the dominant failure modes in PEM device, since it can lead to a non-homogeneous current distribution on the PEM device, and it further accelerate the other failure process. Generally, an electro-thermal finite element modelling of the wirebond substructure is generated by imposing accurate boundary condition to predict the reliability.

For efficient thermal management of PEM structure, an electro-thermal (ET) numerical modelling of the structure is vital in order to identify the critical failure location. Due to the huge computational cost of numerical modelling of PEM structure, most of the academic practitioners tend to model the sub-section of the PEM structure with appropriate boundary conditions and loading to identify the localised effect of the physical process modelled. If the loading varies with time, then many numerical simulations at each discrete time interval are required to extract the physical process modelled, which is computationally expensive. The computational cost further exacerbates if the substructure has a complicated 3D shape, and these require finer numerical discretisation to capture the shape and topology Reduced Order Modelling is widely applied in numerical modelling to reduce the computational cost.

\section{REDUCED ORDER MODELLING}

In the literature, various reduced order models (ROM) have been presented. ROM is described as a compact representation for a full order (high-fidelity) model and captures the physical behaviour of the high-fidelity model. The ROMs approaches can be categorised into three major distinct groups such as (a) surrogate modelling, (b) compact thermal model, and (c) model order reduction which are illustrated in Figure 1. In the surrogate modelling (SM) approach, the response surface technique is combined with design of experiment (DoE) sampling to generate the surrogate model representing the physical process being modelled. SM can be categorised into two distinct groups namely interpolation SM such as the Kriging model [1] and regression SM such as the quadratic polynomial model [2].

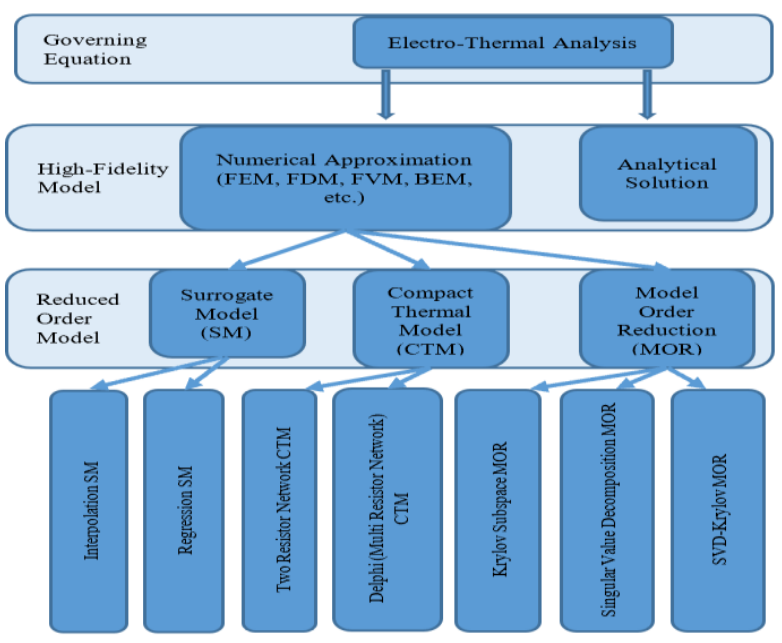

Figure 1: Three major approaches in reduced order modelling

In the compact thermal modelling (CTM) approach the high-fidelity model of the electronic package is replaced with less grid-intensive ROM. This less grid-intensive ROM can preserve the accuracy in predicting the temperatures at key points in the package whilst using far less computational effort. CTM is a simplified representation of a lumped thermal resistor- 
capacitor (RC) network which allows the approximation of the junction temperature and heat fluxes at specific locations. CTM has two approaches such as 2 Resistor network CTM [3] and DELPHI CTM [4].

The final category of ROMs looked at, which forms the basis of this paper, is Model Order Reduction (MOR) approach. Application of the MOR in electro thermal finite element modelling is widely discussed within the literature, see Binion and Chen [5] and Bechtold et al [6]. This study focuses on the application of MOR in electro-thermal finite element modelling to predict the reliability of the wirebond structure of the PEM device in a computationally more efficient way.

\section{METHODOLOGY FOR WIREBOND RELIABILITY PREDICTION BY MOR}

The methodology for predicting the wirebond reliability by utilising MOR is illustrated in Figure 2. For an irregular electric loading in the wire, Joule heating effect generates an irregular temperature distribution in the structure. The time invariant system (TIS) of the wirebond (as described in Section (4.a)) was extracted from finite element code without solving, then MOR algorithms (as described in Section (6.b)) were applied to generate the reduced order TIS. The generalized trapezoidal rule numerical scheme (as described in Section (4.b)) was employed to solve the reduced order TIS. The solution of reduced order TIS was projected back to the original dimension by projection matrix of the MOR algorithms. Then a cycle counting algorithm and a fatigue model (as described in Section (5)) were used to predict the reliability. Next sections address each step in the methodology.

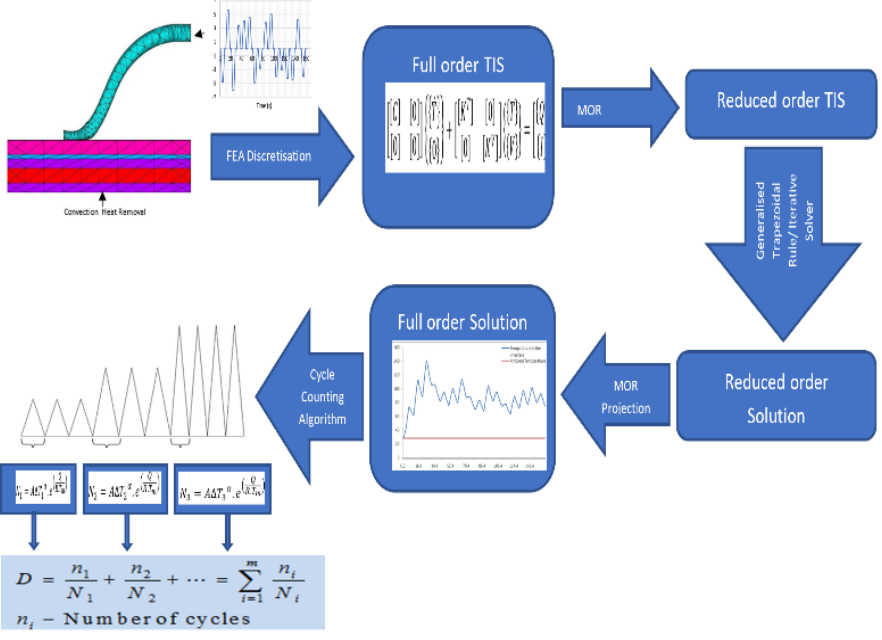

Figure 2: The methodology of the MOR in reliability prediction

\section{WIREBOND ELECTRO-THERMAL FINITE ELEMENT ANALYSIS}

A finite element analysis was undertaken to investigate the residual stress for the prediction of the wirebond wear out failure. The dimensional data of a wirebond (see Lu et al [7]) is used to generate the finite element model, as shown in Figure 3. This model captures a slice of the wire and uses periodic boundary conditions to represent the effect of the array of wires. The mirror plane symmetry of the structure is taken so that only half of the wire and surrounding structure are included in the model to reduce the computational complexity.

Applied electrical loading current to the aluminium wire is illustrated in Figure 4. The ground voltage restriction was imposed on crosscut surfaces of the layers. The thermal

\section{II $27^{\text {th }}$ INTERNATIONAL WORKSHOP}

Thermal Investigations of ICs and Systems )]

convection coefficient of $1000 \mathrm{~W} /\left(\mathrm{m}^{2} . \mathrm{K}\right)$ was imposed on the lower surface of the model to mimic the heatsink surface.

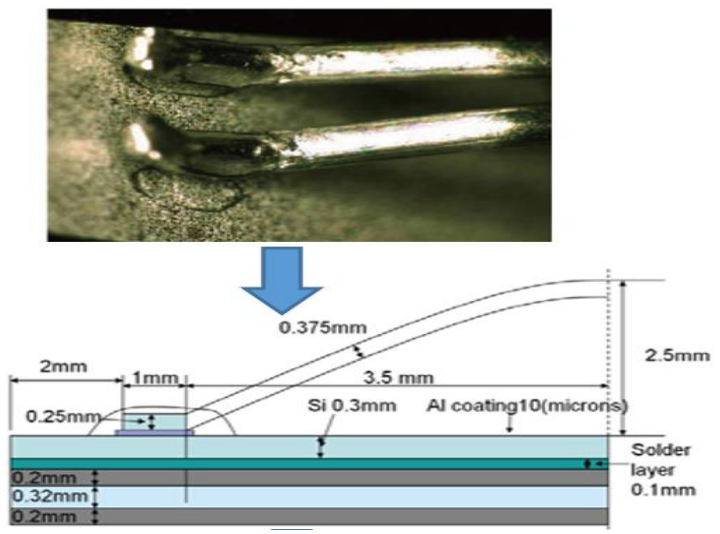

Figure 3: The dimension of the wirebond model

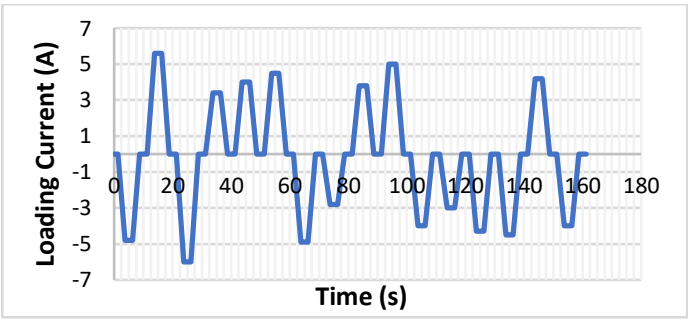

Figure 4: Applied load current through the cross section of the wire [1]

Table 1: Wirebond model material properties for electro-thermal analysis

\begin{tabular}{|l|l|l|l|l|l|}
\hline Parameters & $\begin{array}{l}\text { Si } \\
\text { Chip }\end{array}$ & Copper & AlN & Sn3.5Ag & Aluminium \\
\hline $\begin{array}{l}\text { Resistivity } \\
(\Omega . m)\end{array}$ & 620 & $\begin{array}{l}1.7 \times \\
10^{-8}\end{array}$ & $\begin{array}{l}1 \times \\
10^{14}\end{array}$ & $\begin{array}{l}1.23 \times \\
10^{-7}\end{array}$ & $2.65 \times 10^{-8}$ \\
\hline $\begin{array}{l}\text { Thermal } \\
\text { Conductivity } \\
\text { (W/Mk) }\end{array}$ & 130 & 400 & 160 & 30 & 238 \\
\hline $\begin{array}{l}\text { Specific } \\
\text { Heat } \\
\text { Capacity }\end{array}$ & 700 & 385 & 740 & 219 & 900 \\
\hline $\begin{array}{l}\text { Density } \\
\left(\mathrm{Kg} / \mathrm{m}^{3}\right)\end{array}$ & 2329 & 8960 & 3300 & 7360 & 2700 \\
\hline
\end{tabular}

The material properties of the wirebond structure are as in Table 1. These material data were utilised in the Ansys finite element code to generate the electro-thermal model of the bondwire. The underlying physics involved in the coupled electrothermal finite element numerical model is described in the following section.

\section{a. Underlying Physics}

The conduction currents and the electric flux densities are computed by the following equations.

$$
\{J\}=[\sigma]\{E\}
$$

where $[\sigma]$ - electrical conductivity matrix. $\{E\}$, and $\{J\}$, are electric field $(\mathrm{V} / \mathrm{m})$, and electric current density $\left(\mathrm{A} / \mathrm{m}^{2}\right)$, respectively. For Joule heating, the heat generation term $\ddot{q}$ is derived as

$$
\{\ddot{q}\}=\frac{1}{[\sigma]}|\{J\}|^{2}
$$

The heat transfer equation for the temperature evaluation by Joule heating is defined as

$$
\rho C \frac{\partial T}{\partial t}+\{\nabla \cdot q\}=\{\ddot{q}\}
$$




\section{THERMHIC}

Where $\rho$ - density, C- specific heat, $T$ - temperature, $t$ - time, $\{q\}$ heat flux vector, and $\ddot{q}$ - heat generation rate per unit volume. By Fourier law, heat flux vector and thermal gradients are related by

$$
\{q\}=-[D]\{\nabla T\}
$$

where $[D]$ - conductivity matrix. By combining (3) and (4), heat conduction equation can be derived as

$$
\rho C \frac{\partial T}{\partial t}=\{\nabla \cdot[D]\{\nabla T\}\}+\ddot{q}
$$

The equation for a conducting solid with convection surfaces is defined as

$$
\{q\}^{T}\{\eta\}=\{\eta\}[D]\{\nabla T\}=h_{f}\left(T_{S}-T_{R e f}\right)
$$

Where $h_{f}$ - film coefficient, $T_{s}$ - surface temperature, $T_{R e f}-$ temperature of the surrounding medium, and $\{\eta\}$ - unit outward normal vector. Electro thermal coupling matrix equation is defined as

$$
\left[\begin{array}{cc}
{[C]} & {[0]} \\
{[0]} & {[0]}
\end{array}\right]\left\{\begin{array}{l}
\{\dot{T}\} \\
\{\dot{V}\}
\end{array}\right\}+\left[\begin{array}{cc}
{\left[K^{T}\right]} & {[0]} \\
{[0]} & {\left[K^{V}\right]}
\end{array}\right]\left\{\begin{array}{l}
\{T\} \\
\{V\}
\end{array}\right\}=\left[\begin{array}{c}
\{Q\} \\
\{I\}
\end{array}\right]
$$

where $\{Q\}$ - convection surface vector and heat generation vector, $\left\{K^{T}\right\}$ - thermal conductivity matrix of material and convection surface, $\{C\}$ - thermal specific heat matrix, $\left\{K^{V}\right\}$ electric conductivity matrix, $\{I\}$ - applied nodal electric current vector. The stiffness $\left(\left[\begin{array}{cc}{\left[K^{T}\right]} & {[0]} \\ {[0]} & {\left[K^{V}\right]}\end{array}\right] \in \mathcal{R}^{2 n \times 2 n}\right)$ and damping $\left(\left[\begin{array}{ll}{[C]} & {[0]} \\ {[0]} & {[0]}\end{array}\right] \in \mathcal{R}^{2 n \times 2 n}\right)$ matrices can be extracted from Ansys finite element code without solving as described by Rudnyi et al [8]. The next section addresses the numerical solution process of the time invariant system (TIS) as in Equation (7).

\section{b. $\quad$ Solution of the Time Invariant System}

Various approaches for numerically solving the TIS (Equation (7)) are described in literature [9]. One of the numerical solution approaches for the first order TIS (Equation (7)) is the generalized trapezoidal rule (GTR). The GTR is defined as

$$
\left\{\begin{array}{c}
\left\{T_{n+1}\right\} \\
\left\{V_{n+1}\right\}
\end{array}\right\}=\left\{\begin{array}{c}
\left\{T_{n}\right\} \\
\left\{V_{n}\right\}
\end{array}\right\}+(1-\theta) \Delta t\left\{\begin{array}{c}
\left\{\dot{T}_{n}\right\} \\
\left\{\dot{V}_{n}\right\}
\end{array}\right\}+\theta \Delta t\left\{\begin{array}{c}
\left\{\dot{T}_{n+1}\right\} \\
\left\{\dot{V}_{n+1}\right\}
\end{array}\right\}
$$

where $\theta$ - transient integration parameter (typically in the range of 0.5 and 1$), \Delta t=t_{n+1}-t_{n},\left\{\begin{array}{l}\left\{T_{n}\right\} \\ \left\{V_{n}\right\}\end{array}\right\}$ - the nodal value of temperature vector and voltage vector at time $t_{n}$ and $\left\{\begin{array}{l}\left\{\dot{T}_{n}\right\} \\ \left\{\dot{V}_{n}\right\}\end{array}\right\}$ - the time nodal rate of change of temperature vector and time nodal rate of change of voltage vector at time $t_{n}$ (computed at previous time step). Equation (7) can be rewritten at time $t_{n+1}$ by substituting (8) into it

$$
\begin{aligned}
&\left(\frac{1}{\theta \Delta t}\left[\begin{array}{cc}
{[C]} & {[0]} \\
{[0]} & {[0]}
\end{array}\right]+\left[\begin{array}{cc}
{\left[K^{T}\right]} & {[0]} \\
{[0]} & {\left[K^{V}\right]}
\end{array}\right]\right)\left\{\begin{array}{l}
\left\{T_{n+1}\right\} \\
\left\{V_{n+1}\right\}
\end{array}\right\}=\left[\begin{array}{c}
\{Q\} \\
\{I\}
\end{array}\right]+ \\
& {\left[\begin{array}{ll}
{[C]} & {[0]} \\
{[0]} & {[0]}
\end{array}\right]\left(\frac{1}{\theta \Delta t}\left\{\begin{array}{c}
\left\{T_{n}\right\} \\
\left\{V_{n}\right\}
\end{array}\right\}+\frac{1-\theta}{\theta}\left\{\begin{array}{c}
\left\{\dot{T}_{n}\right\} \\
\left\{\dot{V}_{n}\right\}
\end{array}\right\}\right) } \\
& A\left\{\begin{array}{l}
\left\{T_{n+1}\right\} \\
\left\{V_{n+1}\right\}
\end{array}\right\}=B
\end{aligned}
$$

where $A=\left(\frac{1}{\theta \Delta t}\left[\begin{array}{ll}{[C]} & {[0]} \\ {[0]} & {[0]}\end{array}\right]+\left[\begin{array}{cc}{\left[K^{T}\right]} & {[0]} \\ {[0]} & {\left[K^{V}\right]}\end{array}\right]\right)$, and

$$
B=\left[\begin{array}{c}
\{Q\} \\
\{I\}
\end{array}\right]+\left[\begin{array}{ll}
{[C]} & {[0]} \\
{[0]} & {[0]}
\end{array}\right]\left(\frac{1}{\theta \Delta t}\left\{\begin{array}{l}
\left\{T_{n}\right\} \\
\left\{V_{n}\right\}
\end{array}\right\}+\frac{1-\theta}{\theta}\left\{\begin{array}{l}
\left\{\dot{T}_{n}\right\} \\
\left\{\dot{V}_{n}\right\}
\end{array}\right\}\right)
$$

The equation (10) is a linear system, which can be solved by direct or indirect numerical methods. In Figure 5.a, the

\section{II $27^{\text {th }}$ INTERNATIONAL WORKSHOP}

Thermal Investigations of ICs and Systems I)

numerical solution of TIS (Equation (10)) by GTR is plotted against Ansys simulation results at the intersection point for applied electrical load (Figure 4). Numerical solution by GTR is identical to Ansys solution. The nodal temperature data (Figure (5.a)) is at the wirebond/chip intersection point as illustrated in Figure (5.b)
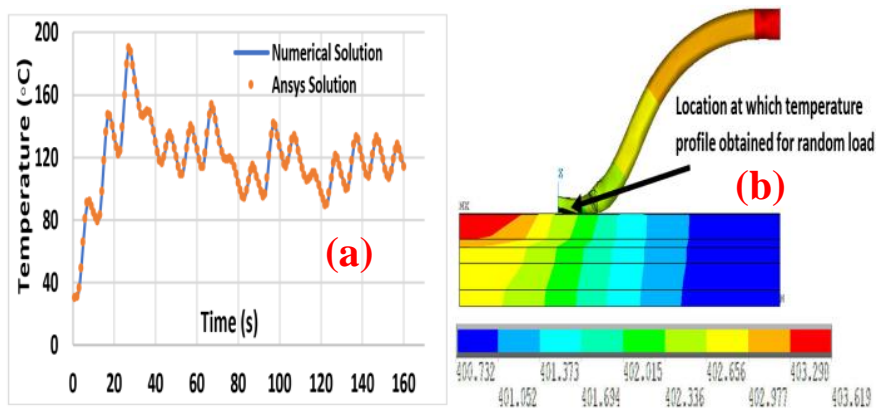

Figure 5:(a) The comparison of numerical solution (Equation (10)) and the Ansys solution of the nodal temperature on the wirebond/chip intersection point (Figure 5.b) versus time (s), (b) Intersection location at which the temperature profile (Figure 5.a) was extracted for current load profile (Figure 4)

\section{RELIABILITY PREDICTION OF THE WIREBOND STRUCTURE}

In order to predict the reliability of the wirebond for power cycling, a fatigue model proposed by Held et al [10] was utilised. The Held's fatigue model depends on the amplitude of the temperature cycle and on the mean value of the thermal cycle by means of Arrhenius term as defined in Equation (11)

$$
N_{f}=A \Delta T^{\alpha} \cdot e^{\left(\frac{Q}{R \cdot T_{m}}\right)}
$$

where $N_{f}$ - the number of cycles to failure by power cycling, $\Delta T$ - the difference between maximum and minimum temperature, $T_{m}$ - the mean temperature in Kelvin, $A, \alpha, Q$ are constants values of $640,-5$, and $7.8 \times 10^{4} \mathrm{Jmol}^{-1}$, respectively. $R$ is the gas constant (8.314 J/mol.K). The fatigue model (Equation (11)) is descriptive only and it does not consider the physical structure of the wirebond or actual failure mechanisms of the wirebond, see [11]. The random load current on the crosscut interface generates random fluctuation temperature on the structure by Joule heating. Generally, the fatigue models such as equation (11) can be applied to cyclic loading, hence a cycle counting algorithm was utilised to extract the cycles.

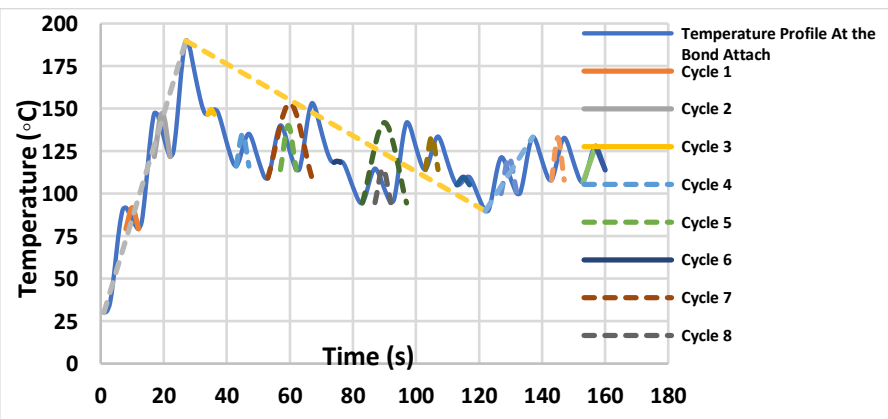

Figure 6: Temperature profile on the heel of the wirebond and the associated cycles extracted by rainflow cycle counting algorithms

Rainflow counting algorithm which was first proposed by Matsuishi and Endo [12] is widely cited in the literature. The Rainflow cycle counting algorithm (RCCA) according to the ASTM E1049 standard was utilised for the temperature profile 


\section{HERN}

(Figure 5.a) of the wirebond. Figure 6 illustrates the plots of the extracted cycles by RCCA.

Summing the damages caused by the cycles extracted from RCCA, a linear damage rule (LDR) can be utilised. LDR is number of significant influences are ignored, and there will be failure prediction errors. The most significant drawback to the linear damage rule is its inability to account for the order of loading. Therefore, the failure prediction by this rule is independent of the load interaction effects that have been observed experimentally. LDR is defined as $D=\sum_{j=1}^{19} \frac{n_{j}}{N_{j}^{f}}$, where $D$ denotes the damage, $n_{j}$ and $N_{j}^{f}$ are the applied number of cycles of operation and total cycles to failure under $j^{\text {th }}$ constant amplitude load level. Tables 2 illustrate the total damage $D$ by LDR for load profile in Figure 4.

Table 2: Total damage D count by LDA

\begin{tabular}{|l|l|l|l|l|}
\hline $\begin{array}{l}\text { Temperatur } \\
\text { e Range } \\
\left(\Delta T\left({ }^{\circ} C\right)\right)\end{array}$ & $\begin{array}{l}\text { Mean } \\
\text { Temperatur } \\
\mathrm{e}\left(T_{m}\left({ }^{\circ} C\right)\right)\end{array}$ & $\begin{array}{l}\text { Number } \\
\text { of } \\
\text { Cycles } \\
\left(n_{j}\right)\end{array}$ & $\begin{array}{l}\text { Number } \\
\text { of Cycles } \\
\text { to Failure } \\
\left(N_{j}^{f}\right)\end{array}$ & $\begin{array}{l}\text { Wear out } \\
\text { Ratio } \\
(W R= \\
\left.\frac{n_{j}}{N_{j}^{f}}\right)\end{array}$ \\
\hline 12.49 & 85.46 & 1 & $4.84 \times 10^{8}$ & $2.065 \times 10^{-9}$ \\
\hline 25.49 & 134.46 & 1 & $5.9 \times 10^{5}$ & $1.695 \times 10^{-6}$ \\
\hline 2.82 & 148.04 & 1 & $1.7 \times 10^{10}$ & $5.884 \times 10^{-11}$ \\
\hline 19.00 & 125.64 & 1 & $4.27 \times 10^{6}$ & $2.344 \times 10^{-7}$ \\
\hline 26.05 & 127.06 & 1 & $8.1 \times 10^{5}$ & $1.235 \times 10^{-6}$ \\
\hline 0.78 & 118.69 & 1 & $5.63 \times 10^{13}$ & $1.776 \times 10^{-14}$ \\
\hline 44.01 & 131.13 & 1 & $4.64 \times 10^{4}$ & $2.153 \times 10^{-5}$ \\
\hline 20.02 & 104.66 & 1 & $1.21 \times 10^{7}$ & $8.252 \times 10^{-8}$ \\
\hline 19.47 & 123.81 & 1 & $4.20 \times 10^{6}$ & $2.381 \times 10^{-7}$ \\
\hline 4.53 & 107.36 & 1 & $1.70 \times 10^{10}$ & $5.866 \times 10^{-11}$ \\
\hline 47.27 & 118.10 & 1 & $7.03 \times 10^{4}$ & $1.422 \times 10^{-5}$ \\
\hline 21.25 & 110.62 & 1 & $6.11 \times 10^{6}$ & $1.636 \times 10^{-7}$ \\
\hline 24.88 & 120.34 & 1 & $1.52 \times 10^{6}$ & $6.586 \times 10^{-7}$ \\
\hline 159.91 & 109.95 & 0.5 & 264 & $1.892 \times 10^{-3}$ \\
\hline 100.17 & 139.83 & 0.5 & 466 & $1.072 \times 10^{-3}$ \\
\hline 43.47 & 111.48 & 0.5 & $1.62 \times 10^{5}$ & $3.093 \times 10^{-6}$ \\
\hline 26.50 & 119.96 & 0.5 & $1.13 \times 10^{6}$ & $4.409 \times 10^{-7}$ \\
\hline 21.17 & 117.30 & 0.5 & $4.10 \times 10^{6}$ & $1.219 \times 10^{-7}$ \\
\hline 14.00 & 120.88 & 0.5 & $2.60 \times 10^{7}$ & $1.922 \times 10^{-8}$ \\
\hline $\begin{array}{l}\text { Total Wear out }(\%) \text { of the wirebond heel structure } \\
\text { for the applied current profile }(\text { Figure } 4)\end{array}$ & & $0.3008 \%$ \\
\hline
\end{tabular}

\section{MODEL ORDER REDUCTION}

The Model Order Reduction (MOR) is a mathematical technique to reduce the dimension of the time invariant system matrices arising from a numerical discretisation of partial differential/integral equations while preserving the character of the input - output relations. The dimensional reduction of the original system introduces an error; nonetheless, this error value should be within an acceptable tolerance for the methods.

The MOR techniques in the literature can be categorised into two main groups namely (a) projection-based moment matching techniques and (b) singular value decomposition (SVD) or Gramian based method as in Figure 7. When an SVD based method is applied to stable systems, it preserves the stability of the system and additionally estimates a priori the bound of the approximation error. However, for large systems, the SVD based MOR techniques are computationally expensive to evaluate and it overshadow the benefit of MOR application to

\section{II $27^{\text {th }}$ INTERNATIONAL WORKSHOP}

Thermal Investigations of ICs and Systems I)

the TIS solution. Moment matching or Krylov subspace methods are based on the moment matching technique, the goal of this techniques is to construct a reduced dimension system that matches a certain number of moments.

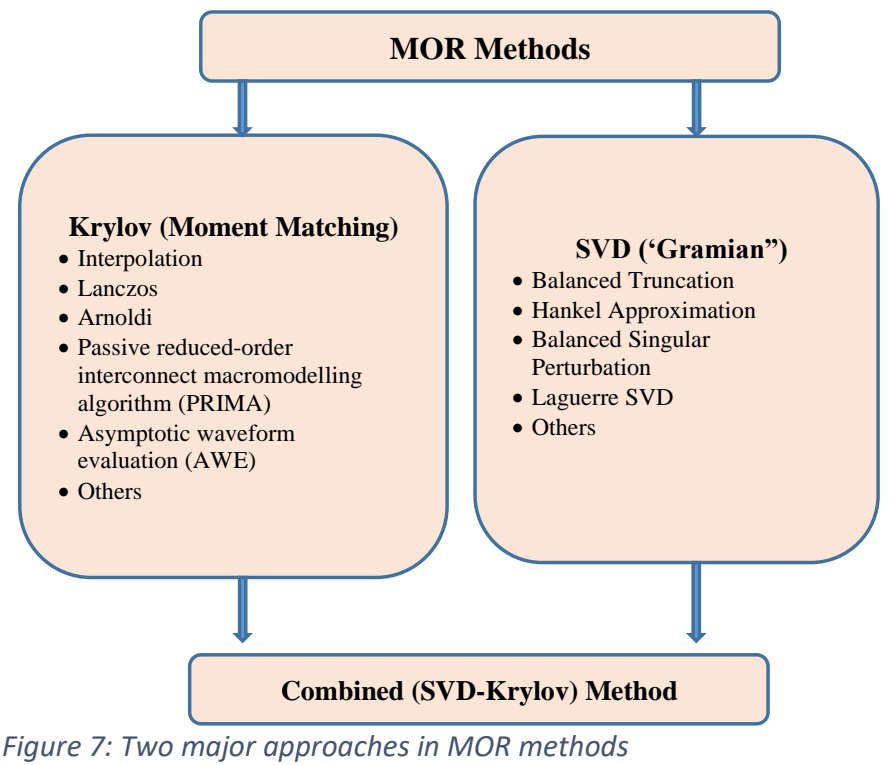

Compared to SVD based methods, moment matching methods are relatively faster to evaluate, however, moment matching methods do not have a priori error bound, hence, this is a disadvantage as the error can become larger than the allowable errors. The TIS (equation (10)) can be written in simplified form as

$$
E \dot{\xi}=A \xi+B
$$

where $E=\left[\begin{array}{cc}{[C]} & {[0]} \\ {[0]} & {[0]}\end{array}\right], A=\left[\begin{array}{cc}{\left[K^{T}\right]} & {[0]} \\ {[0]} & {\left[K^{V}\right]}\end{array}\right], B=\left[\begin{array}{l}\{Q\} \\ \{I\}\end{array}\right]$, and

$\xi=\left\{\begin{array}{l}\{T\} \\ \{V\}\end{array}\right\}$

Laplace transform of the Equation (12) yields,

$s E \xi(s)=A \xi(s)+B(s)$

The transfer function $H(s)$ is defined as

$$
H(s)=(s E-A)^{-1} B
$$

Transfer function (Equation 14) can also be written as

$$
H(s)=\left(s I-E^{-1} A\right)^{-1}\left(E^{-1} B\right)=-\sum_{i=0}^{\infty} m_{i} s^{i}
$$
where $m_{i}=\left(A^{-1} E\right)^{i}\left(A^{-1} B\right)$ is ${ }^{-1} \sum_{i=0} m_{i} s^{i}$ (15) function $H$ by Taylor expansion around $\mathrm{s}=0$. In essence, the moment matching based MOR technique involves choosing the transformation matrix $V$ and projection matrix $W$ in order to transform the compact system into lower dimensional reduced system approximation as below

$$
W^{T} \cdot E V z=W^{T} A V z+W^{T} B(t)
$$

Equation (16) can be written in simplified form as

$$
E_{r} \dot{z}=A_{r} z+B_{r}
$$

where $(W, V) \in \mathcal{R}^{n \times m}, m \ll n . E_{r}, A_{r}$ and $B_{r}$ are reduced matrices of the $E, A$ and $B$.

\section{a. Residual Error By MOR}

The residual error $r$ caused by the MOR is defined as $r=A \xi+$ $B(t)-E \dot{\xi}$, and requirement of the inner product of $r$ with $W$ should be zero $\left(W^{T} r=0\right)$. If $W=V$, then the MOR is named orthogonal projection methods and solution of Equation (12) is generated from the reduced model by following substitution, $\xi \approx$ 


\section{THERMINIC}

$V z$. The project matrices $V$ and $W$ are computed by a MOR method with the MOR projection error of $\varepsilon$ defined as

$$
\varepsilon=\frac{\left\|H(s)-H_{r}(s)\right\|}{\|H(s)\|}
$$

where $H(s)$ and $H_{r}(s)$ are transfer function (Equation (14

)) of full and reduced systems.

\section{b. MOR methods}

Coupled ET analysis setup of the wirebond model resulted in TIS for which various MOR schemes were applied. The most widely used MOR schemes are based on the moment matching (Krylov subspace) technique. One of the drawbacks of the moment matching based MOR is it requires prior knowledge of the number of moments (dimension of the reduced system) to be matched and the expansion point for the transfer function, see Feng et al [13]. I.e., the dimension of the reduced system should be known priory before applying the MOR to full compact system. Expansion point $(s)$ depend on the frequency $(f)$ of the linear time invariant $\operatorname{system}(s=2 \pi j f)$.

The widely used Krylov subspace-based method is Passive Reduced order Interconnect Macro modeling Algorithm (PRIMA) which was originally developed for the RLC circuit system by Odabasioglu et al [14]. Although more expensive in computational cost in forming the projection matrix $V$ of the PRIMA method in comparison with other Krylov based method, PRIMA preserves the stability and passivity of the reduced system. Furthermore, we utilized one of the MOR opensource code namely sssMOR Toolbox [15] in this study. An algorithm named 'Rational Krylov algorithm (RKA) subspace method proposed by Grime [16] which was also used. Another algorithm Iterative Rational Krylov Algorithm (IRKA) proposed by Gugergin et al [17] use iteration to search for an optimal set of shifts (expansion points) in Krylov subspace in order to find a reduced system known as local optimum with respect to $\mathrm{H}_{2}$ norm of the error.

The Arnoldi algorithm (AA) finds the orthogonal vectors of the projector matrices as the columns for projection matrices of Krylov subspaces. In Arnoldi algorithm, the projection matrix $V$ and $W$ are an orthogonal matrix, such that $W^{T} V=I$. Lanczos algorithm (LA) MOR iteratively applies a modified version of Gram Schmidt process to form two pairs of biorthogonal basis matrices for the Krylov subspace, $V$ and $W$. In this study, the Lanczos algorithm by Binion and Chen [5] was utilized. One of the SVD based MOR algorithm Modal Truncation algorithm (MTA) order reduction was also utilized with other Krylov based order reduction algorithms.

Table 3: Model reduction error and time efficiency of the MOR algorithms

\begin{tabular}{|c|l|l|l|}
\hline $\begin{array}{c}\text { MOR } \\
\text { Algorithm }\end{array}$ & $\begin{array}{c}\text { Model } \\
\text { Reduction Error } \\
\text { (Equation (21)) }\end{array}$ & $\begin{array}{c}\text { Time } \\
\text { Efficiency }\end{array}$ & $\begin{array}{c}\text { Total Wear out (\%) } \\
\text { prediction of the } \\
\text { wirebond }\end{array}$ \\
\hline PRIMA & $3.176 \times 10^{-04}$ & 68 & 0.3224 \\
\hline IRKA & $5.545 \times 10^{-9}$ & 65 & 0.2928 \\
\hline AA & $8.762 \times 10^{-9}$ & 62 & 0.1667 \\
\hline LA & $5.09 \times 10^{-04}$ & 59 & 0.3224 \\
\hline RKA & $8.762 \times 10^{-9}$ & 84 & 0.3008 \\
\hline MTA & $7.582 \times 10^{-5}$ & - & 0.3008 \\
\hline
\end{tabular}

$1\left(27^{\text {th }}\right.$ INTERNATIONAL WORKSHOP

Thermal Investigations of ICs and Systems I)

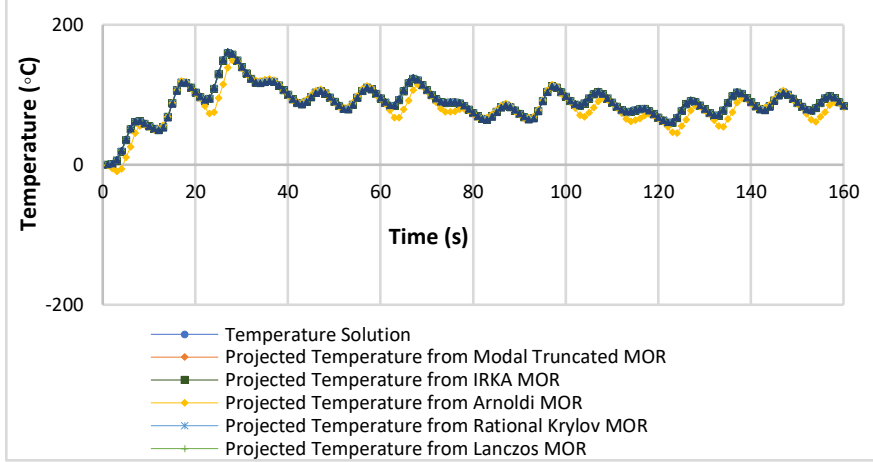

Figure 8: Temperature $\left({ }^{\circ} \mathrm{C}\right)$ at the interface by full order model and temperature projected by MORs.

Table 3 illustrate the projection error and the time efficiency of each MOR algorithms. The error by MOR application is derived by Equation (18). Time efficiency improvement is the ratio between the time taken for full order system iteration and the time taken for reduced order system iteration. The last column indicates the total wear out percentage of the temperature profile at the intersection of the wirebond extracted by each MOR method. For each projected temperature by the MOR algorithms, rainflow cycle counting algorithm and linear damage rule were utilised to predict the total damage as described in Section (5). It can be concluded that in this analysis, the rational Krylov algorithm (RKA) MOR outperforms well in terms of projection error, time efficiency and total wear out $(\%)$ prediction of wirebond in comparison with other MOR algorithms. Furthermore, the total wearout prediction from the temperature projected from the reduced system by RKA MOR is identical to the full order system prediction. Hence applying MOR to a high-fidelity model will indeed reduce the computational complexity by a large factor. Obviously, MOR projection matrices generation requires some additional time, especially for SVD based method, the time required to reduce the system is relatively large. Nevertheless, Once the reduced system is formed then, the solution process is relatively faster in comparison with the full order system. Hence MOR approach is in fact advantage in terms of reducing the computational complexity.

\section{c. $\quad$ Mapping the Projected Temperature Distribution by $M O R$}

The projected temperature by the MOR approximation can be 'mapped' into the finite element solver (Ansys). In Ansys 'external data' option can be utilised to map the projected temperature distribution of the wirebond model. The system matrices extracted from the Ansys EMAT file (see Rudnyi [18]) which consists of the system matrices with reordered indices. Ansys reorders the system obtained from the finite element formation (Equation (10)) for computational efficiency. The reordered system can be reduced by MOR algorithms, solved by an iterative solver and then the solution can be projected back to the original dimension. The projected solution will need to be rearranged against each nodal value and mapped in Ansys. Figure 9 illustrates the mapped results of the temperature distributions projected from MOR algorithms and numerical solution. 


\section{HERMINI}
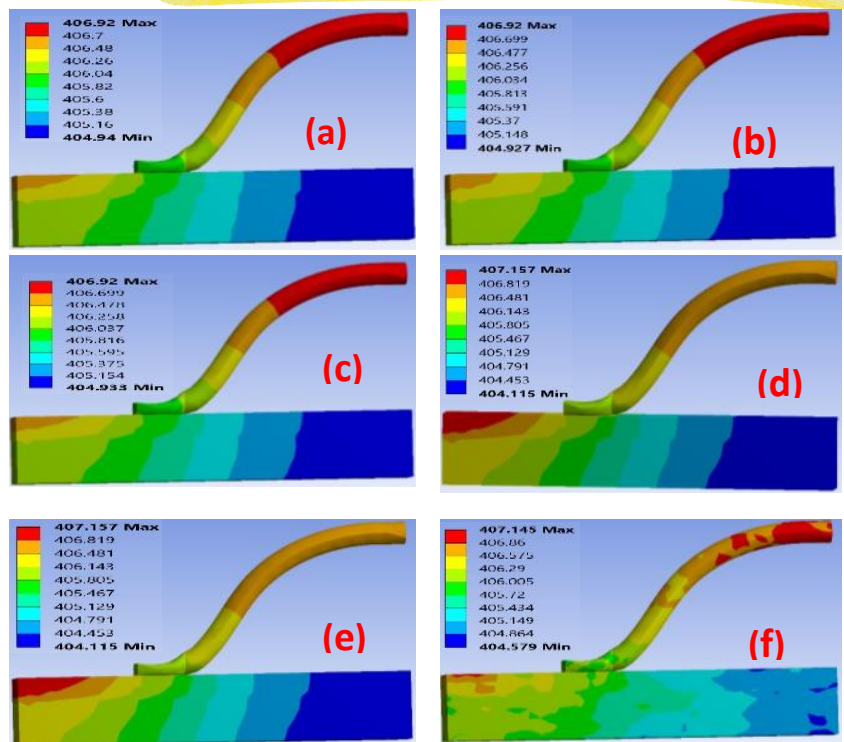

Figure 9: (a) The temperature distribution by the solution of the GTR (Equation (10)) of the wirebond model at 106 seconds of the profile (Figure 4), (b)The temperature distribution by Modal Truncated MOR projection, (c)The temperature distribution by Rational Krylov MOR projection, (d) The temperature distribution by PRIMA MOR projection, (e) The temperature distribution by Lanczos MOR projection, (f)The temperature distribution by IRKA MOR projection

\section{CONCLUSION}

The discretized coupled electro-thermal equations result in a time-invariant system (TIS) of equations that can be solved using a full-field solver such as finite elements. This paper has used a full-field solver such as ANSYS and has implemented a fullfield solver in MATLAB for comparisons with model-order reduction methods. These solvers have been demonstrated for an aluminium wire-bond structure, which is a major reliability risk in power modules due to its electro-thermal behavior. Predictions for temperature have been used to assess fatigue using a fatigue model proposed by Held et al [10]. This fatigue model depends on the temperature range and its mean of the cyclic temperature load, which are calculated using a rainflow cycle counting algorithm. Key conclusions from this work are:

1) The six implemented MOR methods all show benefits in terms of solution times compared to a full-field finite element solver

2) The accuracy of each MOR method in terms of projection error ranges from $5.09 \times 10^{-04}$ for the LA method to $5.545 \times 10^{-9}$ for the IRKA method.

3) The best MOR method in terms of accuracy and computational time is the RKA method which has an accuracy with projection error of $8.762 \times 10^{-9}$ and provides 84 times speed up in solution time compared to a full-field finite element solver.

Hence, it is clear that MOR methods can be used to accurately solve the coupled electro-thermal equations and for power electronic module structures, such as the wire-bond; this provides reliability engineers with a powerful tool for design space exploration.

\section{ACKNOWLEDGMENT}

Martina acknowledges the financial support she received from the University of Greenwich and the partial financial support by Mentor Graphics UK. $1\left(27^{\text {th }}\right.$ INTERNATIONAL WORKSHOP

Thermal Investigations of ICs and Systems I)

\section{REFERENCES}

[1] P. Rajaguru, H. Lu, C. Bailey, "Application of Kriging and radial basis function in power electronic module wirebond structure reliability under various amplitude loading”, International journal of fatigue, vol 45, 2012, pp 61-70

[2] J. Zhang, D. Zhang, "Study of Response Surface Methodology in Thermal Optimization Design of Multichip Modules," IEEE Transactions on Components, Packaging and Manufacturing Technology, vol. 3, no. 12, pp. 2075-2080, 2013

[3] JEDEC Standard, JESD 15-3, July 2008, Two Resistor Compact Thermal Model Guideline, http://www.jedec.org/sites/default/files/docs/JESD 153.pdf

[4] JEDEC Standard, JESD 15-4, October 2008, DELPHI Compact Thermal Model Guideline, http://www.jedec.org/sites/default/files/docs/JESD 154.pdf

[5] D. Binion, X. Chen, "Coupled electrothermal-mechanical analysis for MEMS via model order reduction", Finite Elements in Analysis and Design, 46 (2010) pp 1068-1076.

[6] T. Bechtold, E. B. Rudnyi, and J.G. Korvink," Model Order Reduction for MEMS: Methodology and Computational Environment for ElectroThermal Models". In: Schilders W.H.A., van der Vorst H.A., Rommes J. (eds) Model Order Reduction: Theory, Research Aspects and Applications. Mathematics in Industry (The European Consortium for Mathematics in Industry), vol 13, 2008, Springer, Berlin, Heidelberg

[7] H. Lu, W. Loh, C. Bailey, M. Johnson. "Computer modelling analysis of the globtop's effects on aluminium wirebond reliability". In: 2 nd electronic system integration technology conference, Greenwich, UK, ESTC, 2008. p. 1369-74

[8] E. B. Rudnyi, "MOR for ANSYS, in System-level Modelling of MEMS", edited by Oliver Brand, Gary K. Fedder, Christofer Hierold, Jan G. Korvink, Osamu Tabata, System-level Modelling of MEMS, John Wiley \& Sons, 20 Dec 2012 - Technology \& Engineering

[9] T. J. R. Hughes, "The finite element method: linear static and dynamic finite element analysis", Prentice-Hall, Inc., Englewood Cliffs, NJ, 1987

[10] M. Held, P. Jacob, G. Nicoletti, P. Scacco, and M. H. Poech, "Fast power cycling test for IGBT modules in traction application", Proceedings of Second International Conference on Power Electronics and Drive Systems, Singapore, May 1997

[11] O. Schilling, M. Schäfer, K. Mainka, M. Thoben, F. Sauerland, "Power cycling testing and FE modelling focussed on Al wirebond fatigue in high power IGBT modules", Microelectronic Reliability, 52, 2012, pp 2347 2352

[12] M. Matsuishi, T. Endo "Fatigue of metals subjected to varying stress". In: Paper presented to the Japan society of mechanical engineers, Fukuoka, Japan, March 1968

[13] L. Feng, J. G. Korvink, P. Benner, "A fully adaptive scheme for model order reduction based on moment matching", IEEE Transaction on Component, Packaging and Manufacturing Technology 5(12), 2015.

[14] A. Odabasioglu, M. Celik, L. T. Pileggi, "PRIMA: passive reduced order interconnect macromodeling algorithm", IEEE Transactions on Computer Aided Design of Integrated Circuits \& Systems (TCAD). 17 (8), 1998, pp 645-654

[15] MORLab @ Chair of Automatic Control (2020). sssMOR Toolbox (https://www.mathworks.com/matlabcentral/fileexchange/59169-sssmortoolbox), MATLAB Central File Exchange. Retrieved December 15, 2020.

[16] E. J. Grimme," Krylov Projection Methods for Model Reduction”, Ph.D. thesis, ECE Department, University of Illinois, Urbana-Champaign, 1997

[17] S. Gugercin, A. C. Antoulas and C. Beattie, " $\mathrm{H}_{2}$ model reduction for large-scale linear dynamical systems", SIAM Journal on matrix analysis and applications, 30 (2), 2008, pp 609-638

[18] E. B Rudnyi, MOR for Ansys, in System-level Modelling of MEMS edited by T. Bechtold, G. Schrag, and L. Feng, Willey-Vch, 2013 